\title{
Resource Allocation for Indoor Self-blockage WmWave Device-to-Device Communications
}

\author{
Lei Wang, Member, IEEE, Xiaoting Yu, Jiming Yao, Baoyu Zheng, Senior Member, IEEE, \\ and Guan Gui, Senior Member, IEEE
}

\begin{abstract}
Millimeter wave (mmWave) technology can meet the requirements of large amount of data communications among intelligent devices for indoor scenario. With high requirement of throughput, reducing interference in an enclosed region is still a challenge. For indoor scenario, the human bodies are considered as the main blockages besides conventional blockages. In this paper, we present a new system model by exploiting the selfblockage model to capture the effects of human body, while using multi-ball Line of Sight (LOS) link state model to describe the conventional blockage. The combination of the two components provides a more comprehensive and accurate expression of indoor obstruction. We give a closed expression of coverage rate to analyze the system performance. Simulation results show that the proposed model is highly accurate to describe the distribution of blockages for indoor case. Then, we formulate the resource allocation as an optimization problem under this scenario. The target of this optimization is to achieve the maximum throughput by minimizing interference. To reach this goal, an improved Powell Multi Vertex Coloring algorithm (PMVC) is proposed. The throughput raises apparently when we modify this scheme to put more flows in different time-slots evenly. Numerical experiments show that the improved algorithm can achieve higher system throughput than traditional greedy algorithm and Powell Vertex Coloring (PVC) scheme.
\end{abstract}

Index Terms-mmWave, device-to-device communications, resource allocation, vertex coloring algorithm.

\section{INTRODUCTION}

$\mathbf{R}$ ECENTLY, the indoor networks which provide communications among devices in an enclosed space have attracted more attention. With the increasing amount of mobile devices (e.g., smartphones, tablets) and applications, it is urgent to improve data rate of the indoor communications

This work was supported in part by the Project Funded by the National Science and Technology Major Project of the Ministry of Science and Technology of China under Grant TC190A3WZ-2, the National Natural Science Foundation of China under Grants 61571240 and 61671253, the Qing Lan Project, the Major Projects of the Natural Science Foundation of the Jiangsu Higher Education Institutions under Grant 16KJA510004, the open research fund of National and Local Joint Engineering Laboratory of RF Integration and Micro-Assembly Technology, Nanjing University of Posts and Telecommunications under Grant KFJJ20170305, the Open Research Fund of National Mobile Communications Research Laboratory, Southeast University under Grant 2016D01, the Research Fund of Nanjing University of Posts and Telecommunications under Grant NY218012. (Corresponding authors: Liang Guo and Guan Gui.)

L. Wang, X. Yu, B. Zheng, and G. Gui are with National and Local Joint Engineering Laboratory of RF Integration and Micro-Assembly Technology, Nanjing University of Posts and Telecommunications, Nanjing 210023, China. L. Wang is also with National Mobile Communications Research Laboratory, Southeast University, Nanjing 210096, China. (e-mails: \{wanglei, zby, guiguan\}@njupt.edu.cn,88481473@qq.com)

J. Yao is with Global Energy Interconnection Research Institute Co., Ltd., Beijing 100031, China. (e-mail: yaojiming@geiri.sgcc.com.cn)
[1]-[5]. As an offloading traffic approach, device-to-device (D2D) communications, based on physical proximity device communications, reuses the resource and drastically improves system throughput [6]-[10]. Moreover, it would bring high capacity and seamless transmission in future communication systems [11]-[17]. Due to huge bandwidth available in 60 $\mathrm{GHz}$, mobile devices can achieve multi-gigabit communication services [18], [19]. Although mmWave technology could effectively raise the total system throughput, the severe channel fading problem still exists. Traditional onmi-antenna usually provides massive beams in full coverage azimuth. To reduce pass loss, high gain directional antennas are exploited at mobile devices to obtain high precise transmission in [20][22], intelligently aligning the main beams and nullifying in the orientation of interferences.

However, the capacity for indoor networks is not only related to system bandwidth but also to the density of devices and distribution of obstructions. Thus, the locations of the obstacles or base stations are modeled as Poisson point process (PPP) [23]-[25]. As the density of blockage is vital to the success of D2D transmission, the literature [26] and [27] introduce a three-state path-loss model, including Line of Sight (LOS), non-Line-of-Sight (NLOS) and outage links, but on condition of noise-limited. In [28] and [29], a Boolean rectangle scheme gives a proof that mmWave networks still overmatch ultra high frequency cellular systems in coverage rates and data rates although taking blockages into account. Subsequently, N. Lee et al. proposes a new D2D overlay network, characterized by single-hop networks, but not defines mmWave specific features (e.g., directional antennas, blockage) with clarify [30]. In [31]-[33], the scene is moved into an enclosed area where the human bodies are considered as the main blockages, called self-blockage. It does not take reflections from the ceiling and walls into considerations when modeled. In [34], where the ceilings could highly reflect the signal, leading to more unblocked transmission, a closed form expressions for interference plus noise ratio (SINR) is not given.

When the indoor blockage and interference model is determined, the reasonable resource allocation is urgently required. For an active transmission, a distributed relay selection scheme is proposed in [35], to present a table with details of interference measurements, yet without giving channel unique characteristics. At the same time, vertex-coloring is used to obtain higher throughput in [36], but recomputing weights after coloring enhances the complexity. These works make eminent achievement in blockage modeling and resource allo- 
cation respectively. However, they cannot apply the allocation scheme to the practical blockage case. There is no guarantee on accuracy for indoor resource allocation with simplified model.

There are three major contributions in this paper. First, by combining multi LOS ball link with self-blockage model [37] [38], we improve the interference and blockage model to describe the distributions of obstacles. Second, we derive a closed expression of SINR coverage probability, characterized by body orientation and location. To better analyze system performance, the comparison between SINR analysis and Monte Carlo simulations are illustrated. Third, we formulate the resource allocation as an optimization problem and give an expression to maximize throughput under the condition of the proposed model. However, the optimization under huge interference is a NP-hard problem. For valid counteraction, a new PMVC scheme is presented to deal with interference and increase the total throughput. We compare the PMVC scheme with traditional algorithm, showing that the throughput is improved evenly.

The rest of this paper is organized as follows. The system model is introduced in Section II. In Section III, we establish a SINR model, using a closed expression to analyse coverage probability. While illustrating the theoretical results with Monte Carlo simulations, to depict coverage probability as SINR moves down. The indoor conditions and new PMVC scheme are shown in Section IV. The numerical results of PMVC comparing to other algorithms are given in V. Finally, conclusions and future works are given in Section VI.

\section{SySTEM MODEL}

A smart indoor network is considered, where mobile devices equipping with directional-antennas are served as transmitters or receivers. The directional-antenna characterizes by its main lobe towards the cardinal propagation while other side lobes disperse the energy. Compared with traditional omnidirectional antenna, the adaptive directional-antenna brings the decrease in the additional noise caused by large transmission bandwidth. Meanwhile, it also remedies the increased pathloss at mmWave frequencies. Combined with network geometry, the blockage and interference model is discussed below.

\section{A. Network Model}

Define an infinite region $\Upsilon$ with a reference device pair and several interfering transmitters. The users located in $\Upsilon$ subject to a PPP with the density $\lambda$. These locations are denoted by $g_{k}$. Further, the interferers also can be expressed by the same way $g_{i}$, under a distance of $R_{i}$. As Fig. 1 shown, we assume that each blockage, including human body, is settled to be a circle with the diameter of $\mathcal{D}$. The obstructions associated with reference transmitter are denoted by $B_{k}$, whose azimuth angle to $g_{i}$ is $\theta_{k}$. Similarly, the definition of blockage, which is related to interfering nodes, as $B_{i}$, facing $g_{k}$ with angle $\theta_{i}$.

Assumption 1: To further explore the relations between blockages and interferers, the ring model is utilized to describe the locations of obstacles. When the user holds a mobile device in hand, the transmitter or receiver is bounded to distribute

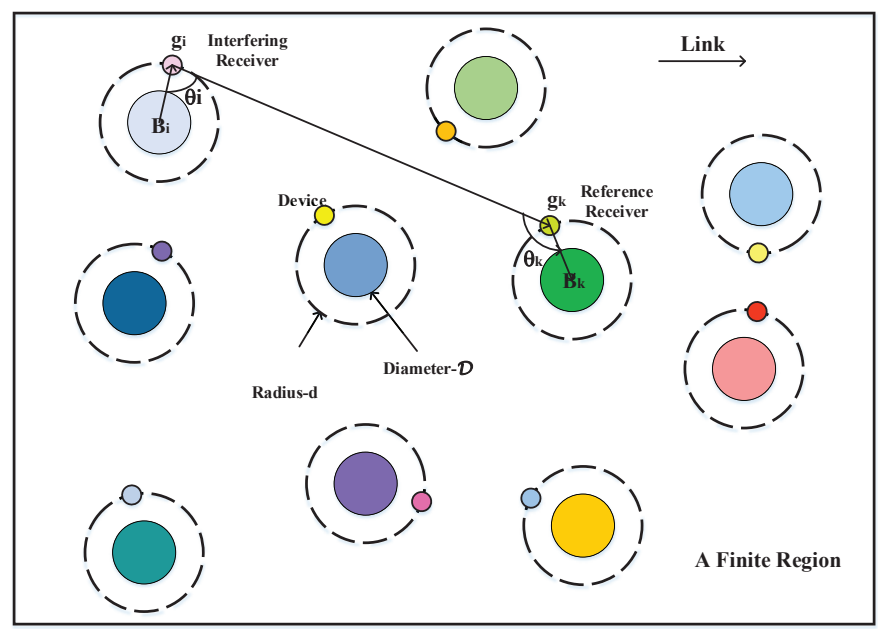

Fig. 1: An illustration of orbital models with the blockages randomly distributed in a finite region, facing $g_{k}$ with $\theta_{i}$ and facing $g_{i}$ with $\theta_{i}$.

around himself. As the planar graph looks like a ring in Fig. 1 , we consider it as a ring model. Hence, the position $g_{k}$ is selected randomly on the circle with radius $d>\mathcal{D} / 2$ centering the $B_{k}$. It is clear that ring model is a valued and inherent part of the self-blockage where body orientation weighs heavily.

\section{B. Signal Model}

As each device is equipped with a directional antenna, the directional beamforming is exploited to steer beams. The antenna gain $G$ characterized by: main beamwidth $\theta^{\prime}$, main lobe gain $\left(G_{f}\right)$ and back lobe gain $\left(G_{b}\right)$ is denoted by:

$$
G=\left\{\begin{array}{cc}
G_{f} G_{f} & \text { w.p. }\left(\theta^{\prime} / \pi\right)^{2} \\
G_{f} G_{b} & \text { w.p. } 2\left(\theta^{\prime} / \pi\right)\left(\pi-\theta^{\prime} / \pi\right) \\
G_{b} G_{b} & \text { w.p. }\left(\left(\pi-\theta^{\prime}\right) / \pi\right)^{2}
\end{array}\right.
$$

We assume that the antenna gain is $G_{f} G_{f}$ under the conditions of perfect alignment of transmitting main lobe and receiving main lobe in the formula above, with probability $\left(\theta^{\prime} / \pi\right)^{2}$. Similarly, if the transmitting main lobe is biased to receiving back lobe, the gain $G_{b} G_{b}$ appears with probability $\left(\left(\pi-\theta^{\prime}\right) / \pi\right)^{2}$. Since there are two cases where the main lobe is shifted to back lobe, the gain $G_{f} G_{b}$ occurs in a probability $2\left(\theta^{\prime} / \pi\right)\left(\left(\pi-\theta^{\prime}\right) / \pi\right)$.

\section{Blockage and Interference Model}

The transmission link is defined either LOS or NLOS. Owing to the high attenuation of mmWave, the link state totally depends on the indoor environment, where blockages scatter all around. Besides the conventional obstacles, the human bodies are the primary blockages for indoor mmWave communications. During transmission between typical reference pairs, $g_{k}$ is not only blocked by $B_{i}$, but also much possible blocked by its own correlative blockage $B_{k}$. In other words, there is even greater likelihood that the transmission link is blocked by the user himself, called self-blockage, which is related to whether $g_{k}$ are straightly facing $g_{i}$. Due to the special indoor mmWave situation, the two assumptions in the following are given for better illustration of key ideas in Fig. 2 . 
Assumption 2: When transmission link falls into a determined cone of angle in space, self-body human blockages happen, as shown in Fig. 2. The parameter $\theta$ is defined as the blocking angle, which is relevant to the width of the human body and its position. Under the assumption that each transmission link path is identically and independently distributed, they arrive with fixed chance $j_{\mathrm{r}}=\theta / 2 \pi$. The constant signal attenuation across the cone is defined in advance. If signal path drops into the angle of blockage cone, there will be an attenuation in received power expressed by a constant factor $\mathcal{F}$. When $\mathcal{F}$ is close to zero, the system is much simplified.

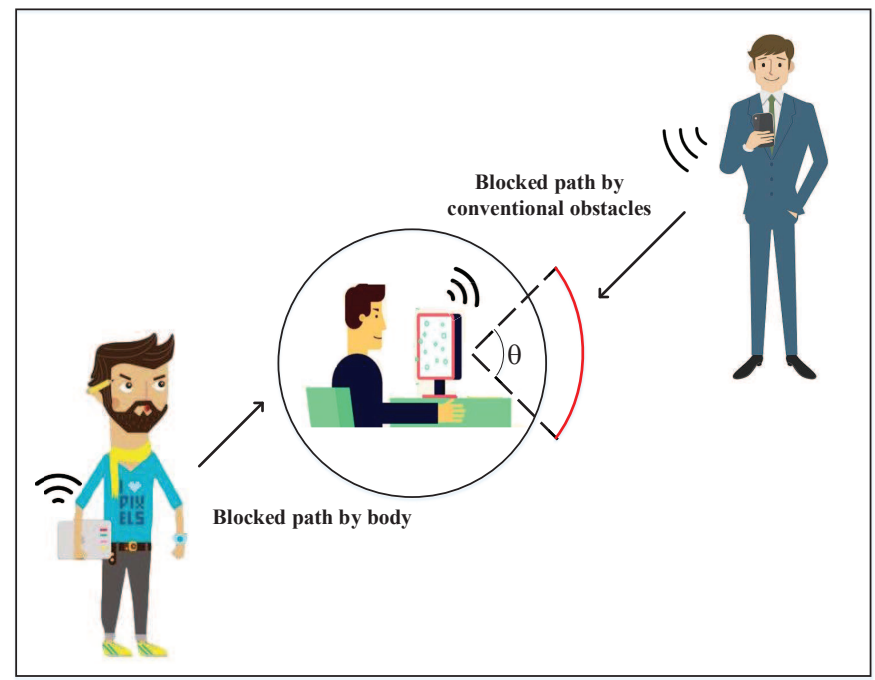

Fig. 2: A blocking cone shows the signal paths which are blocked by human body and conventional obstacles respectively under a condition of indoor case.

Assumption 3: The locations of the blockages still subject to independent point processes. There are two kinds of blockages presumed in this finite region, which includes common obstacles and human body obstructions. That is to say, the communication link would be blocked by conventional blockages or more likely human bodies. For Assumption 3, except for $B_{i}$ and $B_{k}$, we assume signal link also could be blocked by $B_{j}, j \neq i$. The distinct difference between the selfblockage and these ordinary blockages is whether it depends on the locations of $g_{k}$ and $g_{i}$. Actually, the self-blockage happens while $g_{k}$ are not facing towards $g_{i}$. For example, if a user turns his back to the arrival link, the transmission will absolutely be blocked. However, the ordinary blockage has no limited restrictions. Accordingly, this assumption would occur when no signal link is self-blockage and these two cases should be discussed separately.

Assumption 4: The small-scale fading can be obviously neglected for the small-scale fading results in an ignorable impact. The received power is hardly affected while the directional antennas are used in mmWave indoor case. In addition, Nakagami fading is independent for each link, so that the small-scale fading is common to mention. To reduce the complexity, we ignore the changes in channel response, as well as frequency selection.

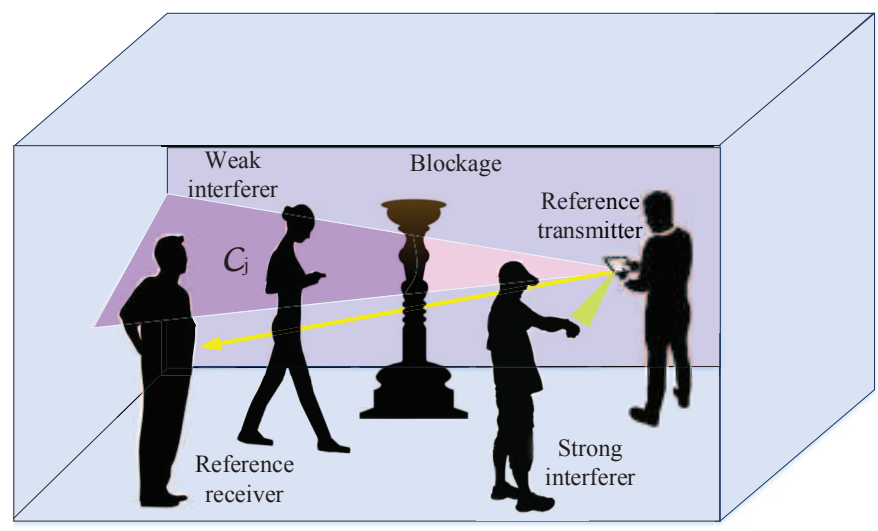

Fig. 3: In an indoor finite region a blocking zone stretched from Fig.1 is exhibited.

If there are blocks $B_{j}, j \neq i$ absent from reference transmitters to receivers, we assume it is strong interference and $g_{i}$ is defined as strong interferer. Otherwise, the link is noted as weak interference with $g_{i}$ being weak interferer. As shown in Fig. 3, on the condition of being no self-blockages during transmission link, once $g_{i}$ blocked by $B_{j}$, the area $\mathcal{C}_{j}$ is assumed to be the blocking zone where any user could not obtain successful transmission. When $g_{i} \in \mathcal{C}_{j}, g_{i}$ is a weak interference. And a blocking zone shown in Fig. 3 is given.

$$
\mathcal{C}_{j}=\left\{\begin{array}{l}
g_{i} \in \Upsilon: \frac{\mathcal{D}}{2}<\left|g_{i}-B_{j}\right| \\
\leq \frac{\mathcal{D}}{4} \sin ^{-1} \frac{\theta_{k}}{2}, \forall j \neq i
\end{array}\right\}
$$

Under this assumption, if $g_{i}$ is a weak interference, it is more likely that there exists blocks between reference pairs. There are $S$ different states assumed to define the blockage model, which include LOS and NLOS. Accordingly, we exploit the multi LOS ball link state and further describe the distribution of indoor obstacles $B_{j}, j \neq i$. The distance from the reference transmitter to reference receiver is divided into $C$ number of LOS link balls from near to far, in order to judge whether $B_{j}, j \neq i$ belongs to this ball. As the radius increases, the ball is approaching the reference receiver. Once $B_{j}, j \neq i$ exists in the ball, the link to be NLOS, means a failure in transmission; otherwise link to be LOS, requires further verification. The $P_{s}(\cdot)$ is noted as the probability of the node's being in state, calculated as:

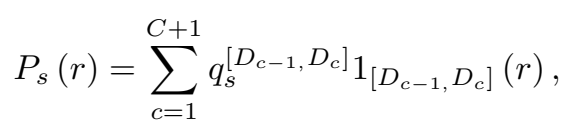

where $\sum_{s \in S} p_{s}(r)=1$ and $C$ indicates the number of circles. When $D_{0}=0$ and $D_{c+1}=+\infty$ are predetermined, $D_{c}$ is supposed to be the radius of the circle $C$. Define an indicator function $1_{\left[D_{c-1}, D_{c}\right]}(r)$ to judge whether $r$ belongs to $[x, y)$, which returns unity if $r \in[x, y) ; 0$ otherwise. The probability $q_{s}^{\left[D_{c-1}, D_{c}\right]}$ implies the link in state $S$ when $r \in\left[D_{c-1}, D_{c}\right)$.

$$
\sum_{s \in S} q_{s}^{\left[D_{0}, D_{1}\right]}(r)=\cdots=\sum_{s \in S} q_{s}^{\left[D_{c-1}, D_{c}\right]}(r)=1
$$

It is obviously that the more balls exist, the more accurate the model is. However, as $B_{j}$ increases, the complexity of analysis also increases. In order to get a balance between these two factors, as shown in (4), a three-ball approximation 
is defined here. The multi ball approximation in (4) can be considered as an expansion of single LOS ball. We decompose the (3) into (4) where $D_{3}>D_{2}>D_{1}>0$. As the radii $D_{c}$ can arbitrarily be any value, the regions emerge for LOS state with different probability. With $\Upsilon$ divided into four regions, we need to approximate the mean link probabilities of different state in (4). The LOS link is finally computed as $p_{L}(r)=\min \{\mathcal{G} / r\}\left(1-e^{\left(-\frac{r}{2 \mathcal{G}}\right)}\right)+e^{\left(-\frac{r}{2 \mathcal{G}}\right)}$, where $\mathcal{G}$ is correlative to the range and the situation of the enclosed region $\Upsilon$. So that we can easily obtain the probability of link in NLOS, denoted as $p_{N}(r)=1-p_{L}(r)$. And the second equation in (4) is an approximation constraint which ensures that the state of these regions is either LOS or NLOS.

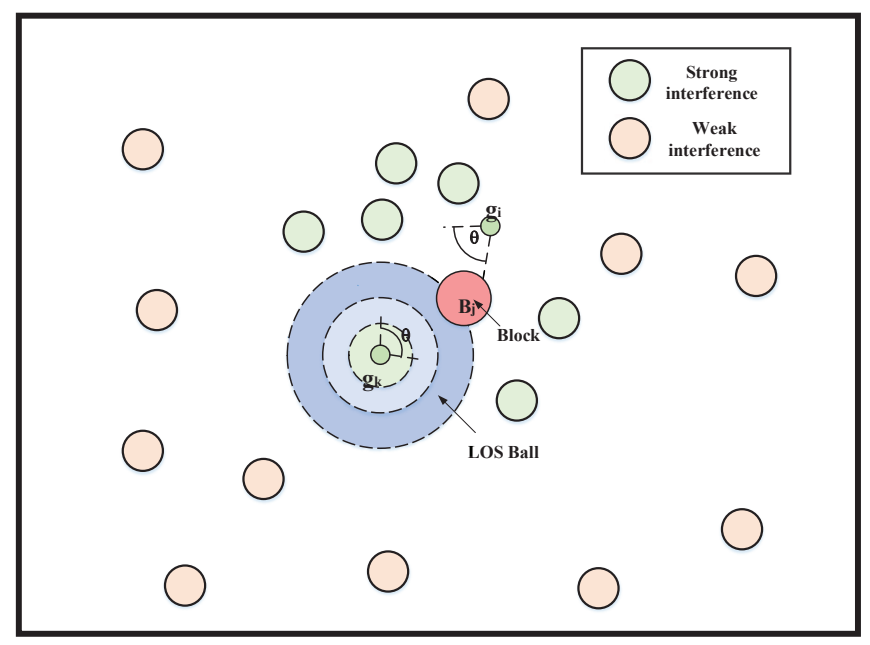

Fig. 4: Plot showing blocking region $B_{i}$ with the fixed threshold $R_{B}$ accompanied with strong interference and weak interference scattering all around. The LOS link balls from the near to far to verify whether this is a LOS link.

Further, in order to determine if there exists $B_{j}, j \neq i$, a region $\Im$ is given. Since $B_{j}, j \neq i$ also defers to PPP, the probability of no user in region $\mathcal{C}_{j}$ is expressed as $e^{-\lambda|\Im|}$. As illustrated in Fig. 4, the actual area of $\Im$ is approximated by:

$$
\Im=\frac{\pi D_{c}^{2}}{2}
$$

Considering the limits of (5), the probability of any user in $\Im$ is obtained by

$$
p_{b}\left(g_{k}\right)=1-e^{-\lambda\left(\frac{\pi D_{c}^{2}}{2}\right)} .
$$

The distance between the interferer and the reference receiver is random. The longer the distance is, the higher probability the block $B_{j}, j \neq i$ exits. The threshold value $R_{B}$ is to give a judgement whether the transmission path is blocked. Whenever the probability $p_{b}\left(g_{k}\right)$ is achieved, the threshold value $R_{B}$ is determined. This is calculated by preserving the average numbers of strong interferers. As once a link is blocked, obviously the interference is weak and we need to abandon. Instead, we hold the average number of strong interferers with the expression

$$
\begin{aligned}
\rho\left(g_{k}\right) & =E\left(\sum_{g_{k} \in \Upsilon} I_{\phi}^{W}\right) \\
& =2 \pi \lambda \int_{g_{k} \in \Upsilon}\left(1-p_{b}\left(g_{k}\right)\right) g_{k} d g_{k} \\
& =2\left(1-e^{-\frac{\pi \lambda D_{c}^{2}}{2}}\right)
\end{aligned}
$$

As the mean number of interferers in a threshold of radius $R_{B}$ is $\lambda \pi R_{B}^{2}$, the value of radius reference to (7) is

$$
R_{B}=\left[\frac{\rho\left(g_{k}\right)}{\lambda \pi}\right]^{0.5} \text {. }
$$

\section{SINR ANAlysis of COVERAge Probability}

The SINR seen from receiver is denoted by

$$
S I N R=\frac{P_{t} M_{0} h_{0} r^{-\alpha_{L}}}{\sigma^{2}+\sum_{i \in \Phi} P_{t} M_{0} h_{i} l(r)},
$$

where $P_{t}$ is transmit power and $M_{0}$ is the antenna gain related to alignment of both main beams. Due to Nakagami fading being used, both $h_{0}$ and $h_{i}$ are defined as normalized Gamma function with random variable $N$. And the noise power delivered by the reference transmitter is denoted as $\sigma^{2}$.

\section{A. Coverage Probability}

A coverage probability denoted by $\beta$ is given when the complementary cumulative distribution function (CCDF) of SINR is greater than a threshold

$$
P_{c}=\{\beta \geq \gamma\} \text {. }
$$

Plugging the quantities in (10), and rearranging it leads to

$$
\begin{aligned}
P_{c} & =\mathrm{P}\left\{\frac{P_{t} M_{0} h_{0} r^{-\alpha_{0}}}{\sigma^{2}+\sum_{i \in \Phi} P_{t} M_{i} h_{i} d_{i}{ }^{-\alpha_{i}}} \geq \gamma\right\} \\
& =\mathrm{P}\left\{h_{0} \geq \frac{\gamma r^{\alpha_{0}}}{P_{t} M_{0}}\left(\sigma^{2}+\sum_{i \in \Phi} \frac{P_{t} M_{i} h_{i}}{d_{i}{ }^{\alpha_{i}}}\right)\right\} \\
& =\mathrm{P}\left\{h_{0} \geq \frac{\gamma r^{\alpha_{0}}}{P_{t} M_{0}}\left(\sigma^{2}+I_{\Phi}\right)\right\},
\end{aligned}
$$

where $I_{\Phi}=I_{\Phi}^{W}+I_{\Phi}^{S}$ indicates the summation of the strong interference and weak interference. Under the circumstance of self-blockage, the effects brought by strong interferer are largely debased. However, due to the self-blockage, signal with a weak interferer is exposed to more weakened link state. The LOS or NLOS signal link is decorated with a passloss exponent $\alpha_{L}$ or $\alpha_{N}$ respectively, and $\alpha_{N}>\alpha_{L}$. As the number of self-blockages is predetermined, the path-loss reference to the features of LOS/NLOS is follows:

$$
l(r)=\left\{\begin{array}{c}
|r|^{-\alpha_{L}} B_{L}^{-s} \\
|r|^{-\alpha_{N}}
\end{array} .\right.
$$

where the upper one occurs if there is a strong interferer, and the other one utilizes an indicator function to distinguish 
the condition of strong interferers. Actually, experiencing a weak interferer, some diffractions and scatterings in $\Upsilon$ are still inevitable. (11) could be further expressed as:

$$
\begin{aligned}
P_{c} & =\mathrm{P}\left\{h_{0} \geq \frac{\gamma r^{\alpha_{0}}}{P_{t} M_{0}}\left(\sigma^{2}+I_{\Phi}\right)\right\} \\
& =1-E\left[\left(1-e^{-\overline{\eta \gamma}\left(\sigma^{2}+\sum_{i \in \Phi} P_{t} M_{i} h_{i} l(r)\right)}\right)^{N}\right] .
\end{aligned}
$$

If we presume $\partial$ to be a normalized gamma random variable and a constant $\Theta>0$, the upper bound of the probability is $P(\partial<\Theta)<\left[1-e^{-\bar{\eta} \Theta}\right]$. Besides, $N$ is the parameter of the gamma fading. Hence $\bar{\eta}=N(N !)^{-1 / N}$ is a gamma random variable used to evaluate $\mathrm{CDF}$. Meanwhile, $\bar{\gamma}=\gamma r_{0}^{\alpha_{L}} / P_{t} M_{0}$ needs to be plugged into the formula above. By using binomial distribution, a new approximation can be obtained:

$$
\begin{aligned}
& P_{c} \approx \sum_{n=1}^{N}(-1)^{n+1}\left(\begin{array}{c}
N \\
n
\end{array}\right) e^{-k N(N !) \frac{-1}{N} \bar{\gamma} \sigma^{2}} \\
& \times E_{\Phi}\left(e^{-k N(N !) \frac{-1}{N} \bar{\gamma} I_{\Phi}^{S}}\right) E_{\Phi}\left(e^{-k N(N !) \frac{-1}{N} \bar{\gamma} I_{\Phi}^{W}}\right) .
\end{aligned}
$$

With the $R_{B}$ computed in (8), the Laplace transformation of the LOS and NLOS fields are denoted by:

$$
\begin{aligned}
& E_{\Phi}\left(e^{-k \bar{\eta} \bar{\gamma} I_{\Phi}^{S}}\right) \\
& =E_{N}\left(E_{g_{i} \in \mathrm{C} j}\left(\left(1-q_{s}\right)+q_{s}\left(j_{\mathrm{r}} \wp^{-N}+\left(1-j_{\mathrm{r}}\right) \wp^{-N}\right)\right)\right) \\
& =E_{N}\left(2 \int_{0}^{D_{c}}\left(\left(1-q_{s}\right) R_{B}^{-2}+\sum_{k=1}^{4} j_{\mathrm{r}_{k}} q_{s} \wp^{-N}\right) \frac{r}{R_{B}^{2}} d r\right) \\
& =e^{-2 \pi \lambda q_{s}\left(\frac{D_{c}^{2}}{2}-\int_{0}^{D_{c}} \sum_{k=1}^{4} j_{\mathrm{r}} \wp^{-N}\right)},
\end{aligned}
$$

$$
\begin{aligned}
& E_{\Phi}\left(e^{-k \overline{\eta \gamma} I_{\Phi}^{W}}\right) \\
& =E_{N}\left(E_{g_{i} \in \Upsilon / \mathrm{C} j}\left(\prod_{i=1}^{N} \wp^{-N}\right)\right) \\
& =e^{-\lambda\left(\kappa \sin ^{-1}\left(1-\frac{\mathcal{D}^{2}}{4 d^{2}}\right)^{0.5} \mathcal{Z}_{1}+\left(1-\kappa \sin ^{-1}\left(1-\frac{\mathcal{D}^{2}}{4 d^{2}}\right)^{0.5} \mathcal{Z}_{2}\right)\right),}
\end{aligned}
$$

where $\wp=1+l k \bar{\gamma} \gamma$ and $G$ exactly depict the configuration of each antenna beam direction, related to the antenna gain (1). To achieve expectation terms of strong interference and weak interference, we assume $\mathcal{P}$ number of strong interferers and $\mathcal{M}$ number of weak interferers respectively. Thus, the $\mathcal{P}$ and $\mathcal{M}$ interferers are Poisson distributed with mean $\lambda \pi R_{B}^{2}$ and $\lambda\left|\Upsilon-\frac{\pi \mathcal{D}^{2}}{4}\right|$. Besides, the distance between strong interferer and reference receiver subjects to the distribution $f(r)=2 r / R_{B}^{2}$. We will obtain the two notations with probability falling into block zone and not falling into block zone. By averaging over $\mathcal{P}$ interferers, the strong interference expectation is computed in (15). Similarly, it is available under the weak interference.

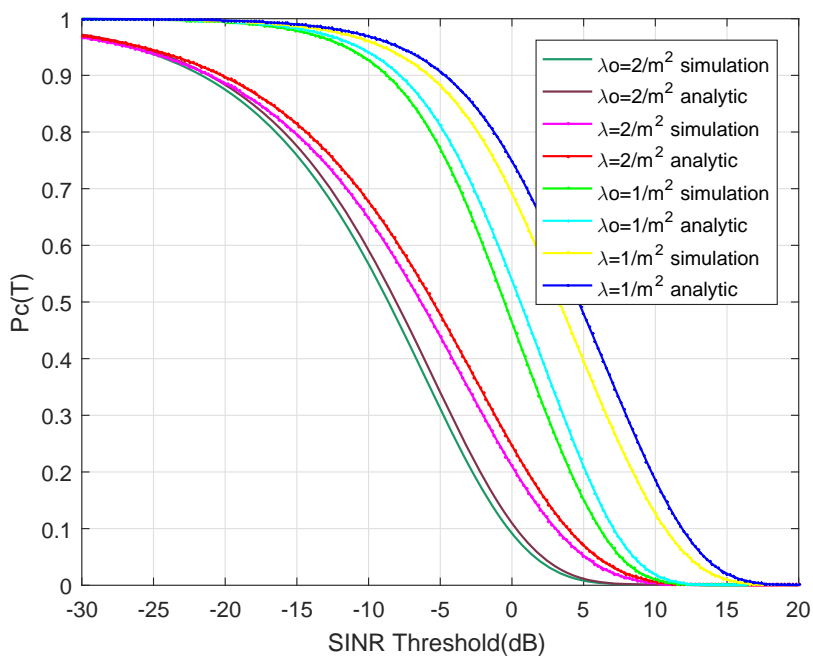

Fig. 5: SINR coverage rate obtained through comparison between Monte Carlo simulation and analytic expression when $\lambda=1$ and $\lambda=2$.

We add the path-loss formula for an exact expression with

$$
\mathcal{Z}_{1}=|v|-\int\left(1+\frac{k \overline{\eta \gamma}}{(|r|)^{-\alpha_{L}}}\right) d r
$$

and

$$
\mathcal{Z}_{2}=|v|-\int\left(1+\frac{k \overline{\eta \gamma}}{(|r|)^{-\alpha_{N}}}\right)^{-N} d r
$$

Each Laplace transformation is simply calculated, except for $\kappa$, where $\kappa=\frac{4 \mathrm{C}_{j}-\pi D_{c}^{2}}{4 \Upsilon-\pi D^{2}}$ depends on antenna gain. The averaged SINR coverage probability can be calculated by adding Eq.(15) and Eq.(16) into Eq.(14). For better transmission capacity we are urgent to capture, the largest $\lambda$ can be easily achieved by presetting SINR threshold and outage $\chi$. It is apparent that once $1-\chi$ appears, the SINR which is larger than a threshold would be received. To achieve this inequality, the distribution of the SINR can be approximated by:

$$
\begin{gathered}
P_{c} \approx \sum_{n=1}^{N}(-1)^{n+1}\left(\begin{array}{c}
N \\
n
\end{array}\right)\left[1-\lambda \varsigma+\lambda^{2} \varsigma^{2}\right] \\
\varsigma=2 \pi U+V \\
U=q_{s}\left(\begin{array}{c}
\left.\frac{D_{c}^{2}}{2}-\int_{0}^{D_{c}} \sum_{k=1}^{4} j_{\mathrm{r}_{k}} \wp^{-N}\right) \\
V=\kappa \mathcal{Q Z}_{1}+\left(1-\kappa \mathcal{Q} \mathcal{Z}_{2}\right) \\
Q=\sin ^{-1}\left(1-\frac{\mathcal{D}^{2}}{4 d^{2}}\right)^{0.5}
\end{array}\right.
\end{gathered}
$$

We use $e^{x} \approx 1+x+x^{2} / 2$ to approximate (15)(16), when $P_{c}$ approaches to 1 . Actually, $U$ and $V$ related to the LOS and NLOS interference respectively. In addition, both $U$ and $V$ can be decomposed further based on gains received from each antenna. In the first equation, the noise, LOS interference and NLOS interference are related to exponential terms. Thus, we can respectively compare the single relative contribution with the total SINR CCDF.

Primarily as SINR changes we present the coverage probability, to show the benefits of the model. With the empirical data given by $\lambda=1$ and $\lambda=2$, the Fig. 5 exhibits SINR 
distributions between self-blockage model and the proposed model. First of all, the errors of the approximation are small. Give a prove that the performance of the system is much more accurate due to the directional antennas and predetermined threshold value $R_{B}$. Actually, with higher density, the reference transmitter is of more risk to experience interferer scattering around. It is clear that the performance of the combined model is better than the original model via comparison.

\section{B. Area Spectral Efficiency}

Area spectral efficiency is the significant metric to evaluate system performance because it can scrutinize the network condition. SINR is denoted by $\Gamma$, the spectral efficiency per channel is

$$
\eta=W_{k} \log _{2}(1+\Gamma) .
$$

where $W_{k}$ is system bandwidth. It is clear that ASE can be obtained through the Monte Carlo simulations and analytical expressions. We can obtain the average spectral efficiency by simulations. Since the interferer randomly distributed in the scenario, we preset a fixed number of interference drawn from a binomial point process (BPP). When a large group of channel realizations is generated, the correlative ASE for each position could be computed exactly. In virtue of the high accuracy, calculations needed to repeat, to obtain the spatial average. With the limit of an infinite number of trials, this method approaches to the precise mean performance nearly. However, the complexity is too high. In contrast, the analytical result just requires to take Rayleigh fading into consideration for the links. The spatially outage probability should be predetermined via measuring the positions of the interferers and the blockages. By confirming the density of interferers and obstacles, we find spatially averaged outage probability. Through this approach, we can easily obtain a closed-form expression for the CCDF of the ASE. According to (20), if $\eta<W_{k} \log _{2}(1+\Gamma)$, we can compute the CCDF spectral efficiency as:

$$
\begin{aligned}
& P_{\eta}[\operatorname{SINR}>\eta] \rightarrow P_{c}\left[\operatorname{SINR}>2^{\frac{\eta}{W_{k}}}-1\right] . \\
& =P_{c}\left(2^{\frac{\eta}{W_{k}}}-1\right)
\end{aligned}
$$

If $X \geq 0, E[X]=\int_{0}^{\infty}(1-F(x)) d x$, the area spectral efficiency could be expressed as:

$$
E[\eta]=\frac{W_{k}}{\ln 2} \int_{0}^{\infty} \frac{P_{c}}{1+\Gamma} d \Gamma,
$$

where $d \eta=d \Gamma / 1 n 2(1+\Gamma)$ varies with $\Gamma=2^{\eta / W_{k}}-1$.

It exists a maximum rate and a minimum rate given by $\beta_{\max }$ and $\beta_{\min }$ during the practice. The modulation order of the distortion and constellation limits in the radio frequency may directly affect maximum rate while the high receiver sensitivity influences the minimum rate. As a result, (22) can be further expressed as:

$$
E[\eta]=\frac{W_{k}}{\ln 2} \int_{\beta_{\min }}^{\beta_{\max }} \frac{P_{c}}{1+\Gamma} d \Gamma .
$$

By averaging the human locations and body orientations,

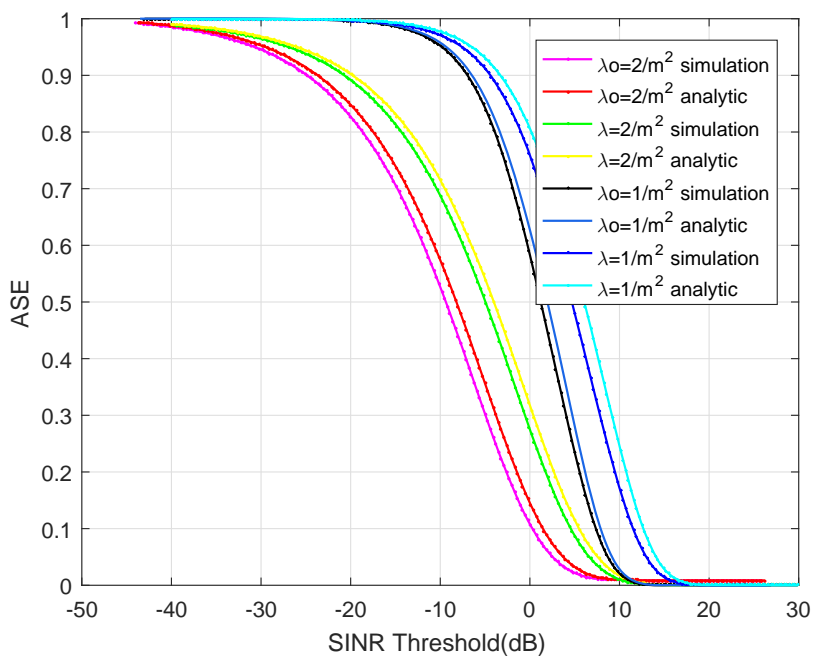

Fig. 6: CCDF of spectral efficiency when $\lambda=1$ and $\lambda=2$ respectively under the comparison between Monte Carlo simulations and analytic results.

we can easily obtain closed-form expression for CCDF of the ASE with different user density. The proposed model and original model are then validated against the results obtained via comparison between analyses and simulations shown in Fig.6. It is clear that the proposed model achieves better performance than self-blockage model.

\section{Optimization of Vertex Coloring Algorithm}

Once blockage modeling is completed, multiple links can transmit simultaneously in such intensive scenarios, which may cause serious interference between links and affect highspeed transmission performance. Therefore, how to design an effective resource allocation algorithm to reduce the interference and improve the network throughput needs to be solved urgently.

Under the blockage and interference model discussed above, in the following, we illustrate the data transmission for indoor D2D communications in $60 \mathrm{GHz}$ network. In this scenario, the focus in indoor resource allocation is the interference between reference pairs. With the accurate interference and blockage model mentioned, the following part gives the interference criteria which refers to threshold value $R_{B}$ and main lobe $\theta^{\prime}$ above. For better tractability, we formulate this resource allocation as an optimization problem while SINR precalculated as a metric utilized to measure the throughput. With some constraints, we maximize throughput to achieve a more sensible scheme. As the total throughput, suffering from the interference, is a NP-hard problem, vertex coloring is assumed to settle the conflicts. By using vertex coloring, the flows which have conflicts during transmission are not in the same time slot.

\section{Indoor condition}

Utilizing Shannon capacity, the accessible transmission data rate is given by:

$$
R_{k}=\alpha W_{k} \log _{2}(1+S I N R),
$$


where $\alpha$ is the data rate loss during the transmission. As each link is independently distributed, the pass-loss exponent varies with receivers. For example, a NLOS link is desired and passloss is defined as $\alpha_{N}$. The throughput of the network can be evaluated as:

$$
R_{\text {sum }}=\sum_{k=1}^{n} R_{k} .
$$

To further raise the throughput $R_{\text {sum }}$, the summation of $R_{k}$ is maximized to achieve a superior allocation scheme. According to (25), we formulate the optimization problem as:

$$
\begin{gathered}
\max _{\left\{I_{\phi}\right\}} \quad \sum_{k=1}^{n} R_{k}, \\
\text { s.t. } \quad S I N R \geq \gamma, \\
0<k \leq n, \\
\sum_{k=1}^{n} P_{r, k}=P_{t} G\left(\frac{\lambda}{4 \pi}\right)^{2}\left(\frac{1}{r}\right)^{n} \leq P_{\max }, \\
\frac{R_{k}}{P_{r, k}} \geq \varepsilon,
\end{gathered}
$$

where the constraint (26b) implies the received SINR must be greater than a threshold, and the constraint (26c) gives a power budget baseline. The constraint (26d) where $P_{r, k}$ is received power at user $k$, rules the fixed time slots since they are limited during propagation. In addition, the constraint (26e) enforces the proportion between achievable throughput and power assumption to be greater than an energy lower bound $\varepsilon$.

It is clear that the optimization in (26) is a resource scheduling problem. The objective is to reduce interference when the threshold is satisfied. Thus, a interference graph is required to describe the situations for indoor scenario. It is a typical vertex cover problem. In the mathematical discipline, a vertex cover problem can be formulated as a half-integral linear program which is one of Karp's 21 NP-hard problems. Owing to the complexity, we exploit coloring vertex scheme to solve this problem. Before coloring, the indoor conflict conditions are required to externalize in details. During propagation, there are two kinds of conflicts defined, including primary conflict and secondary conflict. Apparently, when two links are from different directions, they could not be allocated in the same time-slot. In other words, the common node cannot transmit and receive simultaneously as shown in Fig.7(c), which is known as primary conflict. As Fig.7(d) shows, the interfering nodes confound the reference transmitters and receivers under a threshold range, called secondary conflict. To capture the characteristics of conflicts, the normalized main lobe pattern function is formulated as:

$$
g\left(\theta^{\prime}\right)=\frac{G_{f f}\left(\theta^{\prime}\right)}{G_{\max }} .
$$

According to this, being in beamwidth returns $g\left(\theta^{\prime}\right)=1$; otherwise $g\left(\theta^{\prime}\right)=0$ received. There are two conditions satisfied by the non-interfering simultaneous transmission: (1) whether the correlative receivers are in the beamwidth; (2) whether the correlative receivers are in the short-range of the threshold area with being in beamwidth. Therefore, $R_{B}$ is con-

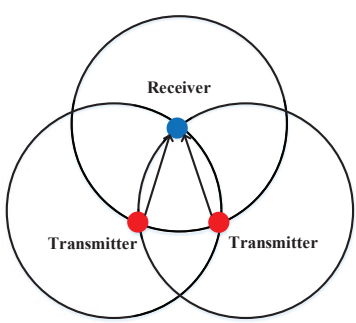

(a) Conflict between transmitter and transmitter

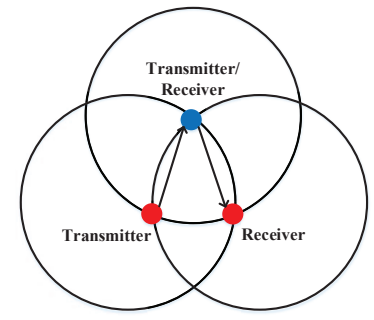

(c) Conflict between transmitter and receiver

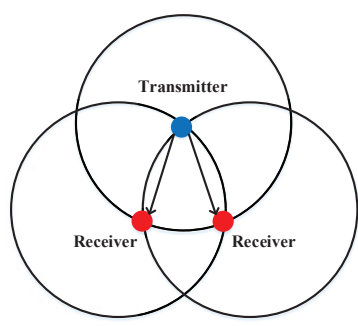

(b) Conflict between receiver and receiver

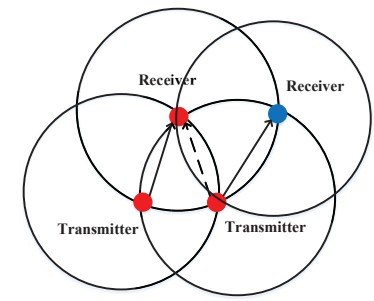

(d) Conflict among transmitter, receiver and transmitter
Fig. 7: Conflicts for indoor transmission.

sidered as the threshold value to judge whether $g_{i}$ is a strong interference or not. While receivers being out the beamwidth $\left|g_{k}-g_{i}\right| \leq R_{B}, g_{i}$ is a strong interference; otherwise not. However, a node locates in the beamwidth of the reference transmitter, not being the range of $R_{B}$, and it still isn't an interference. As the interference criterion defined, there is a secondary conflict when reference transmitter communicates with reference receiver, facing strong interference within the beamwidth in the same slot.

Therefore, by exploiting the limited restrictions mentioned above, the $k \times k$ adjacency matrix due to indoor transmission situation could be achieved where $k$ represents total active flows. Compared to the traditional serial scheme, concurrent transmission may suffer from the possible interference from other transmitters.

$$
\text { AdjacencyMatrix }=\left[\begin{array}{ccccccc}
0 & 1 & 1 & 0 & 0 & 0 & 0 \\
1 & 0 & 1 & 0 & 1 & 1 & 0 \\
1 & 1 & 0 & 1 & 0 & 0 & 1 \\
0 & 0 & 1 & 0 & 0 & 0 & 1 \\
0 & 1 & 0 & 0 & 0 & 0 & 0 \\
0 & 0 & 0 & 0 & 0 & 0 & 1 \\
0 & 0 & 1 & 1 & 0 & 1 & 0
\end{array}\right] .
$$

From (28), the $7 \times 7$ adjacency matrix contains 1 s and 0 s and each row or column represents a flow where 1 implies a conflict. In addition, we could utilize the vertex coloring to allocate the time slot source to each transmitter when getting an adjacency matrix. In order to avoid interference and allocate time slots reasonably, the method of vertex coloring defines each vertex a flow and each color a resource block (time slot). If there is a conflict between two flows, we draw a line between the corresponding vertices. Based on the principle of minimum coloring, it colors neighbouring vertex (linked with each other) with different colors distinctly.

Accordingly, we utilize PVC algorithm based on maximum priority to allocate time slots, which means that the more 

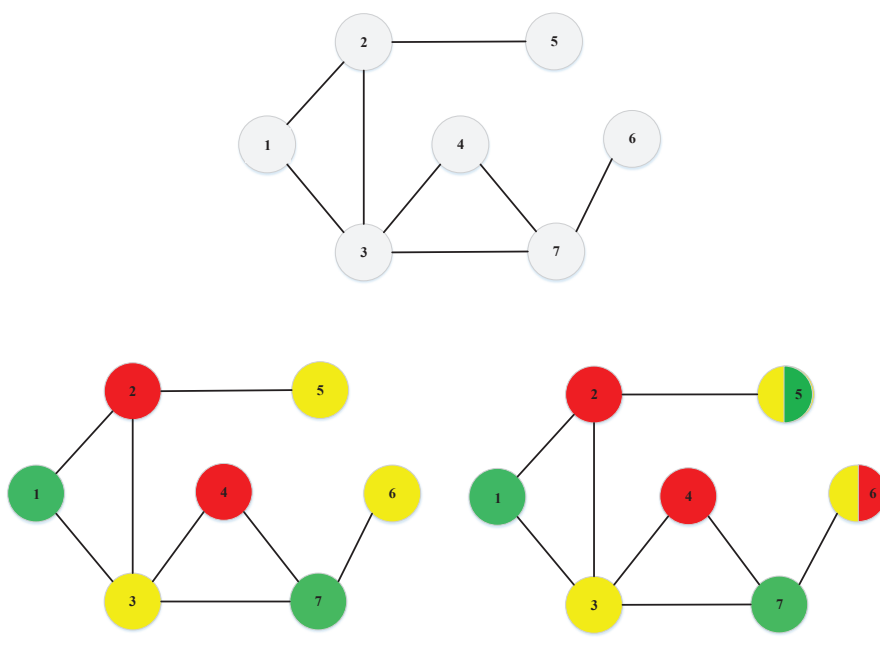

$1,7 \quad 2,4,3,5,6$

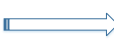

$1,5,72,4,63,5,6$

Fig. 8: The schemes of PVC coloring and PMVC coloring are achieved by adjacency matrix.

edges a vertex has, the more preferentially it is colored. Convincingly, it is of same basic principle with the indoor time slot allocation that a flow with more conflicts needs to be allocated with a high priority. Because of this characteristic, the weights do not demand recalculations. Thus, exploiting PVC scheme should highly according to the indoor resource blockage allocation.

\section{E. Optimization of Vertex Coloring Algorithm}

However, the initial PVC Algorithm even could handle the conflicts among flows while the low efficiency still exists and actually becomes the bottleneck of original approach. Because it is of high susceptibility that each time slot is allocated randomly. Therefore the allocation is full of uncertainty and randomness, so that not all time slots are active. That is to say, some time slots are allocated with more flows, increasing the probability of encountering more conflicts while some others are not. What is more, the time slot is a rare resource and requires to be taken full advantages to maximize the throughput. Consequently, an optimization of PVC Algorithm based on maximum priority is proposed.

As shown in Fig. 8 , the vertex with the most edges is colored in priority. After coloring all the vertices, we add all rows of adjacency matrix to judge the color which is least used and most used, respectively denoted as $\operatorname{Min}(\mathrm{k})$ and $\operatorname{Max}(\mathrm{k})$. Then, the vertices colored by $\operatorname{Min}(\mathrm{k})$ are required to be found next. We sort these least colored vertices by subscript and take the smallest one first. To capture the conflict conditions of the vertex, we define a new matrix $\mathrm{C}$. With $\mathrm{C}$, the vertex with Min(k) could be judged whether are propitious to be added with color $\operatorname{Max}(\mathrm{k})$. Afterwards determine the new $\operatorname{Min}(\mathrm{k})$ and $\operatorname{Max}(\mathrm{k})$. When the number of each color returns to average, the algorithm terminates. The intrinsic principle of multiple coloring for a vertex is similar to the traditional PVC.

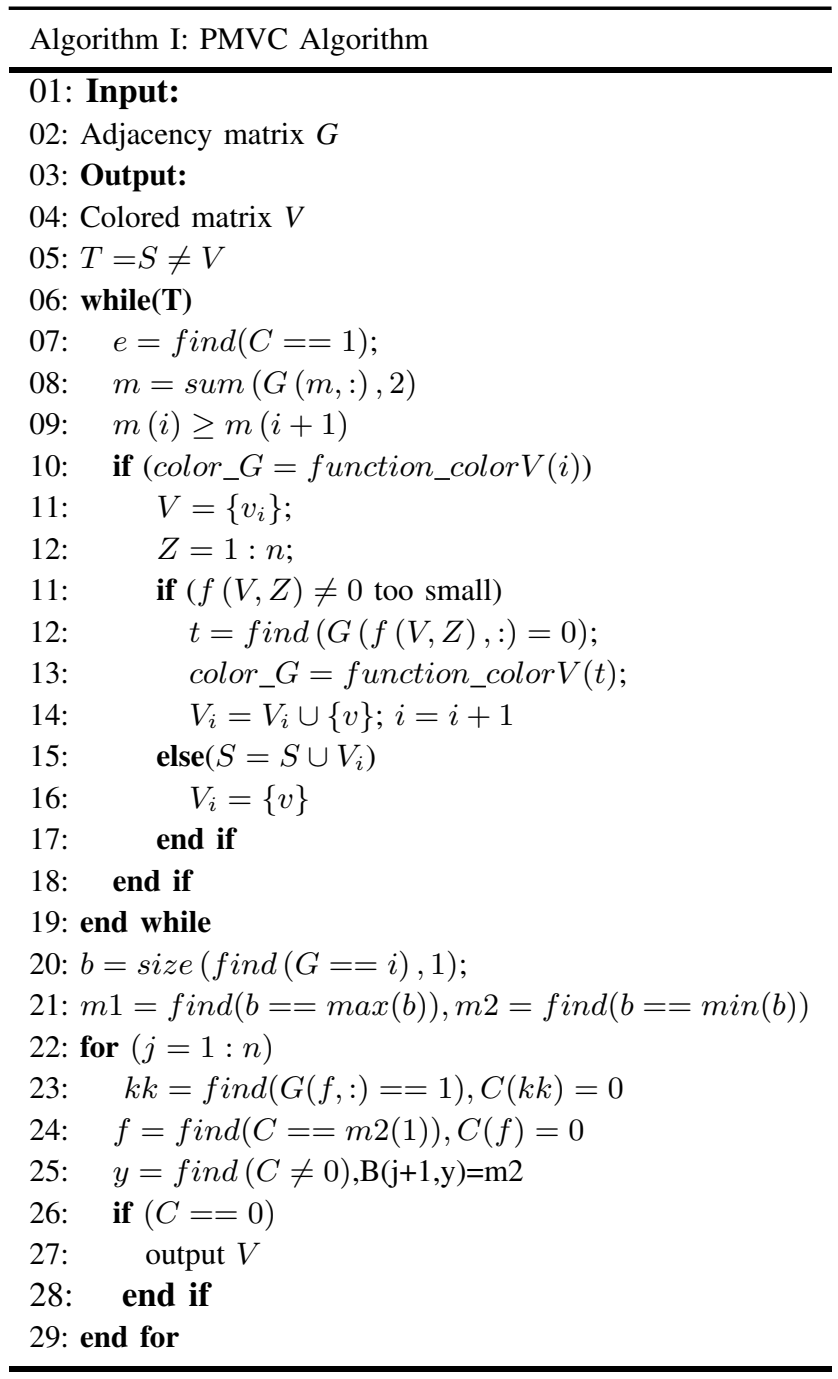

\section{NUMERICAL RESULTS}

TABLE I: Evaluation Notations

\begin{tabular}{|c|c|}
\hline Parameter & Value \\
\hline$\alpha_{L}$ & 2 \\
\hline$\alpha_{N}$ & 4 \\
\hline$\sigma^{2}$ & -100 \\
\hline$N, m, M$ & $7,0.125,8$ \\
\hline$\theta$ & $\frac{\pi}{6}$ \\
\hline$P_{t}$ & $1 \mathrm{w}$ \\
\hline
\end{tabular}

In this section, the comparison between greedy method and modified transmission allocation algorithms are provided. Table 1 lists parameter values used in the simulation.

To further validate the performance of algorithm, with $P T=\lambda \log 2(1+S I N R) P_{c}\{S I N R>\gamma\}$, the throughput is achieved by changing the distance from reference transmitter to reference receiver. The Fig. 9 shows that when the theoretical results are provided to be as a kind of reference, the method we have exploited is compared to the traditional greedy algorithm. It is clear that the performance in throughput of greedy algorithm is far worse than the algorithm we proposed. 


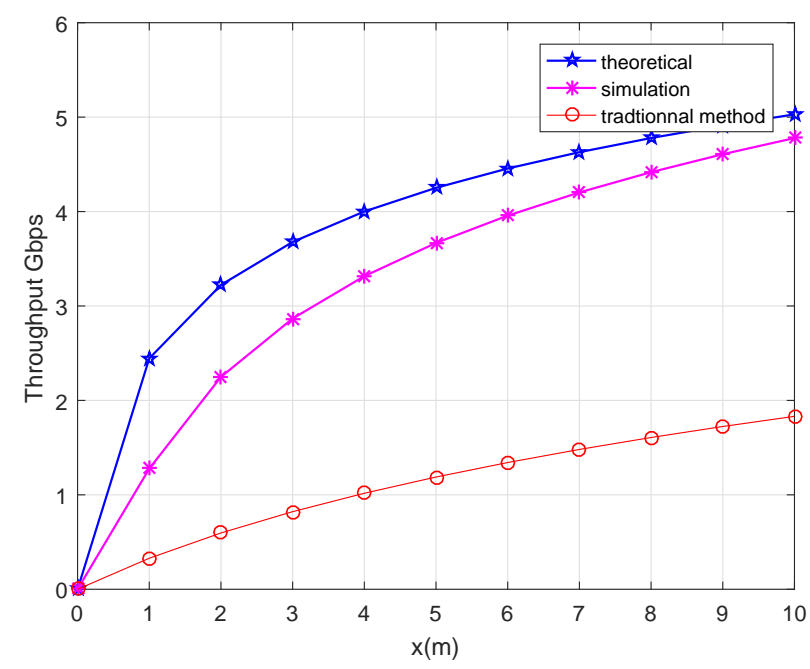

Fig. 9: Theoretical throughput vs simulation throughput vs traditional Greedy method, which proves that PMVC scheme achieves more better throughput.

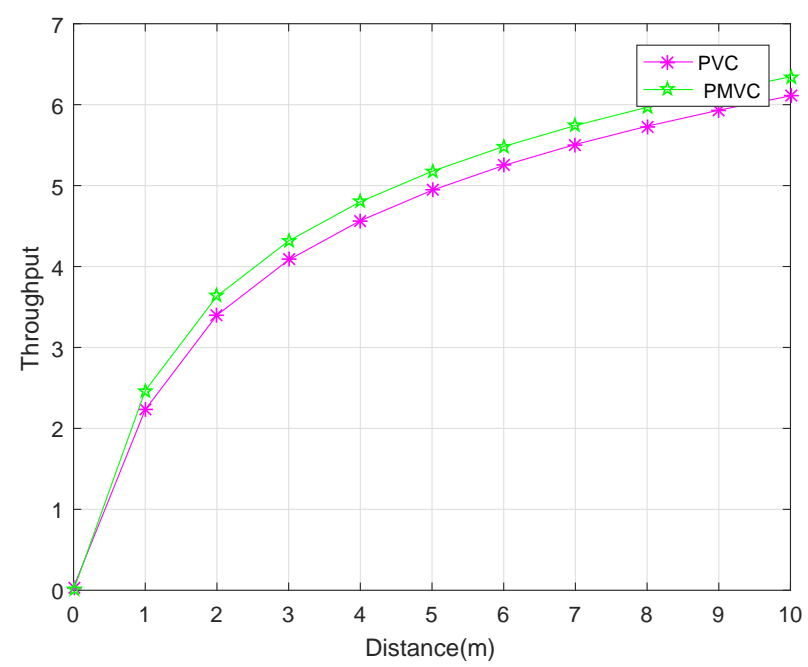

Fig. 10: Throughput comparison between PMVC and PVC illustrates that the former scheme overmatches the later algorithm as the distance increases.

The throughput we have obtained from PMVC scheme is more closer to theoretical throughput. However, there is a huge gap between results from greedy and analytical throughput.

Besides, we evaluate PMVC Algorithm with increasing distance, to compare with PVC scheme. As shown in Fig. 10, PMVC is superior to PVC for PVC just has realized avoiding interference in the same time slot but would cause some time slots allocated with a large amount of flows while the others not. However, except for evading interference, PMVC scheme averages the flows in each slot, nearly leading to no difference in flow per slot. Thus, the variance is largely less than conventional greedy scheme. Then while beamwidth is studied, the total throughput of the active flows is given in Fig. 11. For indoor communication and resource block allocation, with the beamwidth increasing, it implies that the coverage of each signal has expanded along with the beamwidth's increase. Hence, the interference the reference transmitter would face is rising, resulting in the chance where flows could

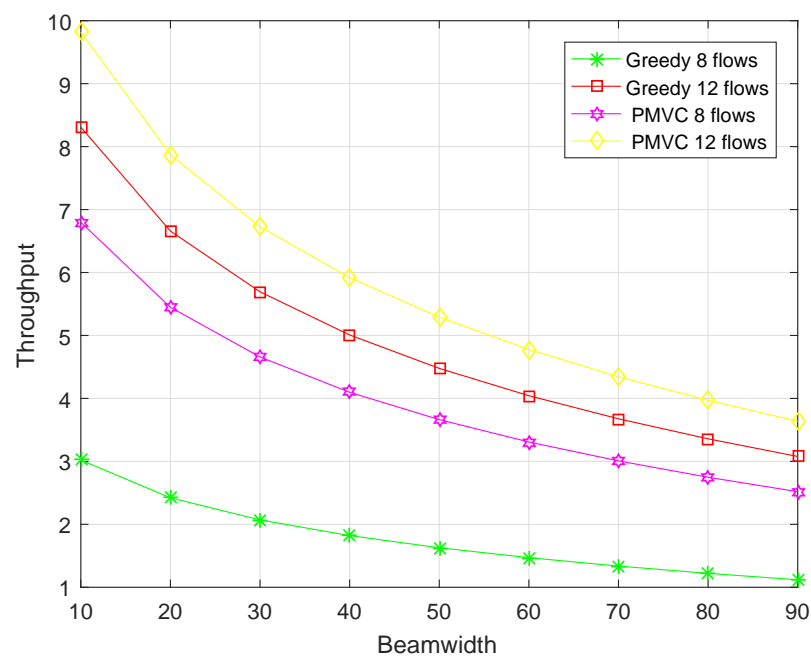

Fig. 11: Throughput comparison between Greedy and PMVC when the number of flows equals to 8 and 12 respectively. As beamwidth increases, PMVC enables more flows to be transmitted in a time slot.

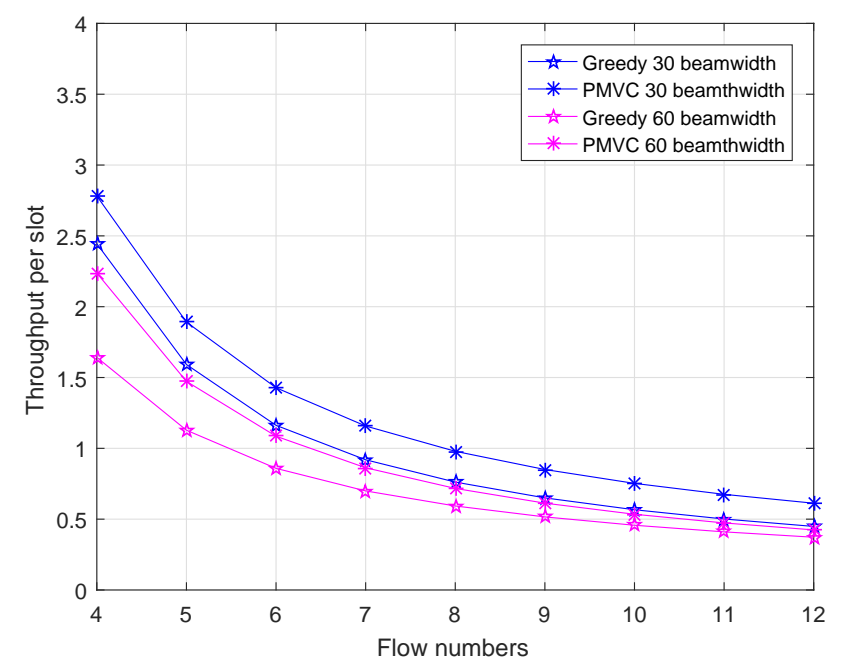

Fig. 12: Throughput per slot against flows when the beamwidth respectively equals to 30 and 60 degree. Also, the decline of rate means that the reference pairs are experiencing more conflicts.

be transmitted in a same time-slot reducing. Under different flows, the throughput drops in different rates, which means the more flows are, the much faster it descends.

Fig. 12 shows flow throughput per slot against increasing number of active flows with beamwidth equal to 30 and 60 degree. As flow numbers rise, the flow throughput per slot decreases. However, it can be seen that PMVC still provides better results than the Greedy algorithm. Accordingly, PMVC scheme can support more data transmission under the circumstance of the same flows. Because there are more flows allocated in a same slot as far as possible when we implement PMVC. Thus, the probability that resource block is allocated unevenly is reduced.

\section{CONCLUSION}

In this paper, we have proposed a system model to characterize the objects and human bodies for indoor scenario, 
integrating multi LOS ball with self-blockage model. To better describe the characteristics of mmWave signal propagation, we assume the Nakagami fading to be independent on each kind of link, characterized by the path-loss exponent. A closed approximation expression of SINR can be given afterwards. The proposed model enables us to estimate system performance which shows a good system performance by comparing the simulation and analysis. When the interference and blockage model is determined, we discuss about the indoor transmission conditions. A maximum expression is given for capturing higher throughput, which leads to a NP-hard problem. To address this optimization problem, we propose a PMVC algorithm to enhance the system throughput, reducing the interference simultaneously. Compared with traditional Greedy algorithm and PVC scheme, the PMVC we proposed achieves much better performance in throughput. In future, we are going to consider the times that the mmWave reflects from the walls and ceiling and define the intensity of the transmission link according to the times. It will help us further explore the relations of strong and weak interferers.

\section{REFERENCES}

[1] B. Ma, H. Shah-Mansouri and V. W. S. Wong, "Multimedia Content Delivery in Millimeter Wave Home Networks," in IEEE Transactions on Wireless Communications, vol. 15, no. 7, pp. 4826-4838, Jul. 2016.

[2] L. Zhou, D. Wu, Z. Dong, and X. Li, "When Collaboration Hugs Intelligence: Content Delivery over Ultra-Dense Networks," IEEE Communications Magazine, vol. 55, no. 12, pp. 91-95, Dec. 2017.

[3] C. Ma, M. Ding, H. Chen, Z. Lin, G. Mao, Y. Liang, and B. Vucetic, "Socially Aware Caching Strategy in Device-to-Device Communication Networks," IEEE Transactions on Vehicular Technology, vol. 67, no. 5, pp. 4615-4629, May 2018.

[4] B. Li, W. Guo, Y. Liang, C. An, and C. Zhao, "Asynchronous Device Detection for Cognitive Device-to-Device Communications," IEEE Transactions on Wireless Communications vol. 17, no. 4, pp. 2443-2456, Apr. 2018.

[5] Y. Pei, and Y. Liang, "Resource Allocation for Device-to-Device Communications Overlaying Two-Way Cellular Networks," IEEE Transactions on Wireless Communications, vol. 12, no. 7, pp. 3611-3621, Jul. 2013.

[6] A. Zhang, J. Chen, R. Hu, Y. Qian, "SeDS: Secure Data Sharing Strategy for D2D Communication in LTE-Advanced Networks," IEEE Transactions on Vehicular Technology, vol. PP, no. 99, pp. 2659-2672, Apr. 2016.

[7] Z. Zhou, J. Gong, Y. He, Y. Zhang, "Software Defined Machineto-Machine Communication for Smart Energy Management," IEEE Communications Magazine, vol. 55, no. 10, pp. 52-60, Oct. 2017.

[8] Z. Zhou, Y. Guo, Y. He, X. Zhao, W. Bazzi, "Access control and resource allocation for M2M communications in industrial automation," IEEE Transactions on Industrial Informatics, vol. 15, no. 5, pp. 3093-3103, May. 2019.

[9] Z. Zhou, M. Dong, K. Ota, G. Wang, L. T. Yang, "Energy-efficient resource allocation for D2D communications underlaying cloud-RANbased LTE-A networks," IEEE Internet of Things Journal, vol. 3, no. 3, pp. 428-438, Jun. 2016.

[10] D. Wu, J. Wang, R. Q. Hu, Y. Cai, and L. Zhou, "Energy-efficient resource sharing for mobile device-to-device multimedia communications," IEEE Transactions on Vehicular Technology, vol. 10, no. 5 pp. 2093-2103, Jun. 2014.

[11] D. Wu, L. Zhou, Y. Cai, R. Q. Hu, and Y. Qian, "The role of mobility for D2D communications in LTE-advanced networks: Energyvs. bandwidth-efficiency," IEEE Wireless Communications Magazine, vol. 21, no. 2, pp. 66-71, 2014.

[12] L. Zhou, R. Q. Hu, Y. Qian and H. H. Chen, "Energy-Spectrum Efficiency Tradeoff for Video Streaming over Mobile Ad Hoc Networks," in IEEE Journal on Selected Areas in Communications, vol. 31, no. 5, pp. 981-991, May 2013.

[13] L. Zhou, "Mobile Device-to-Device Video Distribution: Theory and Application," in ACM Transactions on Multimedia Computing Communications and Applications, vol. 12, no. 3, pp. 1253-1271, 2015.
[14] M. Liu, T. Song and G. Gui, "'Deep cognitive perspective: Resource allocation for NOMA based heterogeneous IoT with imperfect SIC," IEEE Internet of Things Journal, vol. 6, no. 2, pp. 2885-2894, Feb. 2019.

[15] M. Liu, J. Yang, and G. Gui, "DSF-NOMA: UAV-assisted emergency communication technology in a heterogeneous internet of things," IEEE Internet of Things Journal, vol. 6, no. 3, pp. 5508-5519, Jun. 2019.

[16] N. Kato et al., "Optimizing space-air-ground integrated networks by artificial intelligence," IEEE Wireless Communications, to be published, doi: 10.1109/MWC.2018.1800365.

[17] G. Gui, H. Sari, and E. Biglieri, "A new definition of fairness for nonorthogonal multiple access," IEEE Communications Letters, vol. 23, no. 7, pp. 1267-1271, Jul. 2019.

[18] L. Zhou, D. Wu, J. Chen and Z. Dong, "Greening the Smart Cities: Energy-Efficient Massive Content Delivery via D2D Communications," IEEE Transactions on Industrial Informatics, vol. 14, no. 4, pp. 16261634, April 2018.

[19] P. Liu, M. Di Renzo and A. Springer, "Line-of-Sight Spatial Modulation for Indoor mmWave Communication at $60 \mathrm{GHz}$," in IEEE Transactions on Wireless Communications, vol. 15, no. 11, pp. 7373-7389, Nov. 2016.

[20] T. Bai and R. W. Heath, "Coverage and Rate Analysis for MillimeterWave Cellular Networks," in IEEE Transactions on Wireless Communications, vol. 14, no. 2, pp. 1100-1114, Feb. 2015.

[21] M. D. Renzo, "Computational stochastic geometryCon system-level modeling, simulation, performance evaluation, optimization, and experimental validation of $5 \mathrm{~g}$ wireless communication networks," International Conference on Communications, Management and Telecommunications (ComManTel), DaNang, 2015, pp. 1-2.

[22] A. Thornburg, T. Bai and R. W. Heath, "MmWave ad hoc network coverage and capacity," IEEE International Conference on Communications (ICC), London, 2015, pp. 1310-1315.

[23] M. Di Renzo, "Stochastic Geometry Modeling and Analysis of MultiTier Millimeter Wave Cellular Networks," IEEE Transactions on Wireless Communications, vol. 14, no. 9, pp. 5038-5057, Sept. 2015.

[24] A. Thornburg, T. Bai and R. W. Heath, "Performance Analysis of Outdoor mmWave Ad Hoc Networks," in IEEE Transactions on Signal Processing, vol. 64, no. 15, pp. 4065-4079, Aug. 2016.

[25] T. Bai and R. W. Heath, "Analysis of self-body blocking effects in millimeter wave cellular networks," Asilomar Conference on Signals, Systems and Computers, Pacific Grove, CA, 2014, pp. 1921-1925.

[26] T. Bai, R. Vaze and R. W. Heath, "Analysis of Blockage Effects on Urban Cellular Networks," IEEE Transactions on Wireless Communications, vol. 13, no. 9, pp. 5070-5083, Sept. 2014.

[27] M. Di Renzo, "Stochastic geometry modeling and performance evaluation of mmWave cellular communications," IEEE International Conference on Communications (ICC), London, 2015, pp. 5992-5997.

[28] J. G. Andrews, T. Bai, M. N. Kulkarni, A. Alkhateeb, A. K. Gupta and R. W. Heath, "Modeling and Analyzing Millimeter Wave Cellular Systems," IEEE Transactions on Communications, vol. 65, no. 1, pp. 403-430, Jan. 2017.

[29] M. Di Renzo, W. Lu and P. Guan, "The Intensity Matching Approach: A Tractable Stochastic Geometry Approximation to System-Level Analysis of Cellular Networks," IEEE Transactions on Wireless Communications, vol. 15 , no. 9 , pp. 5963-5983, Sept. 2016.

[30] N. Lee, X. Lin, J. G. Andrews and R. W. Heath, "Power Control for D2D Underlaid Cellular Networks: Modeling, Algorithms, and Analysis," IEEE Journal on Selected Areas in Communications, vol. 33, no. 1, pp. 1-13, Jan. 2015

[31] K. Venugopal and R. W. Heath, "Millimeter Wave Networked Wearables in Dense Indoor Environments," IEEE Access, vol. 4, pp. 1205-1221, 2016.

[32] K. Venugopal, M. C. Valenti and R. W. Heath, "Analysis of millimeter wave networked wearables in crowded environments," Asilomar Conference on Signals, Systems and Computers, Pacific Grove, CA, 2015, pp. 872-876.

[33] G. George, K. Venugopal, A. Lozano and R. W. Heath, "Enclosed mmWave Wearable Networks: Feasibility and Performance," IEEE Transactions on Wireless Communications, vol. 16, no. 4, pp. 23002313, April 2017.

[34] K. Venugopal and R. W. Heath, "Location based performance model for indoor mmWave wearable communication," IEEE International Conference on Communications(ICC), Kuala Lumpur, 2016, pp. 1-6.

[35] W. Ur Rehman, T. Salam and X. Tao, "Receiver based distributed relay selection scheme for 60-GHz networks," IEEE Annual International Symposium on Personal, Indoor, and Mobile Radio Communication (PIMRC), Washington DC, 2014, pp. 1585-1590. 
[36] W. ur Rehman, T. Salam and X. Tao, "Vertex multi-coloring scheduling algorithm for concurrent transmission in 60-GHz networks," IEEE Global Communications Conference (GLOBECOM), Austin, TX, 2014, pp. 4751-4757.

[37] K. Venugopal, M. C. Valenti and R. W. Heath, "Device-to-Device Millimeter Wave Communications: Interference, Coverage, Rate, and Finite Topologies," IEEE Transactions on Wireless Communications, vol. 15, no. 9, pp. 6175-6188, Sept. 2016.

[38] M. Abouelseoud and G. Charlton, "The Effect of Human Blockage on the Performance of Millimeter-Wave Access Link for Outdoor Coverage," IEEE Vehicular Technology Conference(VTC Spring), Dresden, 2013, pp. 1-5. 